\title{
Diagnosis and diagnostic tests for fibromyalgia (syndrome)
}

\author{
W. Häuser ${ }^{1,2}$, F. Wolfe ${ }^{3}$ \\ ${ }^{1}$ Department Internal Medicine I, Klinikum Saarbrücken, Germany; \\ ${ }^{2}$ Department of Psychosomatic Medicine and Psychotherapy, Technische Universität München, Germany; \\ ${ }^{3}$ National Data Bank for Rheumatic Diseases, Wichita, Kansas and University of Kansas School of Medicine, \\ Wichita, KS, USA
}

\section{SUMMARY}

Objectives: To present diagnostic criteria for the clinical diagnosis of fibromyalgia syndrome (FMS) and to offer a scheme for diagnostic work-up in clinical practice.

Methods: Narrative review of the literature, consensus documents by the American College of Rheumatology (ACR), evidence-based interdisciplinary German guidelines on the diagnosis and management of FMS.

Results: The ACR 1990 classification criteria emphasized tender points and widespread pain as the key features of FMS. In 2010, the ACR proposed preliminary diagnostic criteria for fibromyalgia that abandoned the tender point count and placed increased emphasis of patient symptoms. A later modification of the ACR 2010 criteria for use in surveys employed a self-report questionnaire (Fibromyalgia Survey Questionnaire FSQ) to assess patient symptoms. The FSQ can be used to assist physician's diagnosis of FMS.

We recommend a stepwise diagnostic work-up of patients with chronic widespread pain (CWP) in primary care: Complete medical history including medication, complete medical examination, basic laboratory tests to screen for inflammatory or endocrinology diseases, referral to specialists only in case of suspected somatic diseases, assessment of limitations of daily functioning, screening for other functional somatic symptoms and mental disorders, and referring to mental health specialists in case of mental disorder.

Conclusions: The diagnosis of FMS is easy in most patients with CWP and does not ordinarily require a rheumatologist. A rheumatologist's expertise might be needed to exclude difficult to diagnose or concomitant inflammatory rheumatic diseases. In the presence of mental illness referral to a mental health specialist for evaluation is recommended.

Key words: Fibromyalgia syndrome, diagnosis, differential diagnosis.

Reumatismo, 2012; 64 (4): 194-205

\section{INTRODUCTION}

$\mathrm{F}$ ibromyalgia (FM)/fibromyalgia syndrome (FMS) is still a controversial condition, because of social and community consequences, as well as disputes about etiology and pathogenesis $(1,2)$. The aims of the present paper are as follows:

- to give an overview on the different validated diagnostic criteria of FMS;

- to point out some open questions in diagnosing FMS;

- to outline a diagnostic work-up for patients with fibromyalgia type symptoms in primary care; Winfried Häuser

Klinik Innere Gedizin Winterberg

D-66119 Saarbrücken, Germany E-mail: whaeuser@klinikum-saarbruecken.de
- to discuss the role of rheumatologists and mental health specialists in the diagnostic process.

\section{METHODS}

We performed a narrative review of the literature on diagnosis of FMS. Our recommendations are based on consensus documents by the American College of Rheumatology (ACR) and by the evidencebased interdisciplinary German guidelines on the diagnosis and management of FMS. American College of Rheumatology (ACR) 1990 criteria

The number of tender points necessary for FM-diagnosis was investigated in the 1990 FM-criteria study (3). To manage examiner heterogeneity, training sessions were undertaken in order to have the 22 study physicians examining patients in the same way. In addition, it was settled that $4 \mathrm{~kg}$ be the 
amount of force exerted by the palpating finger or thumb. In unpublished data from the criteria study, we noted that prior to the training session different examiners used substantially different amounts of force, but that even though examiner variance decreased during the study it was still quite noticeable. That is, even among trained experts there was considerable variability in the performance of the tender point examination. With this as background, a consortium of investigators undertook a criteria study that would ultimately lead to the promulgation of the 1990 ACR classification criteria for FM. A team of 22 rheumatologists served as volunteer investigators. Each contributed from their practice 10 patients with FM, 10 with FM and rheumatoid arthritis, osteoarthritis or axial pain syndromes, 10 patients who did not have FM but other rheumatic conditions involving pain (e.g. osteoarthritis, low back pain), and the last 10 who were patients with rheumatoid arthritis, osteoarthritis or axial pain syndromes without FM. Each patient underwent a tender point examination that included many tender point sites, comprising those known not to be sensitive to pressure. In addition, patients were evaluated for different symptoms of FM, including those of the Yunus criteria (4). Investigators underwent a training session so that the tender point examination was performed the same way by each investigator.

\section{The preliminary ACR 2010 criteria}

These were developed in an US multicenter study of 829 previously diagnosed FM-patients and controls using physician physical and interview examinations by 10 rheumatologists, including a widespread pain index (WPI), a measure of the number of painful body regions. Random forest and recursive partitioning analyses were used to guide the development of a case definition of fibromyalgia, to develop criteria, and to construct a symptom severity (SS) scale (5).

\section{The Fibromyalgia Survey Diagnostic} Criteria (FSDC): these were established in a longitudinal study of patients of the $\mathrm{Na}$ - tional Data Bank for Rheumatic Diseases (6) with 729 patients previously diagnosed with FM, 845 with osteoarthritis (OA) or with other noninflammatory rheumatic conditions, 439 with systemic lupus erythematosus (SLE), and 5210 with rheumatoid arthritis (RA). Since most of the ACR 2010 items can be obtained by self-administration, the preliminary ACR 2010 criteria were slightly modified to make possible a complete self-administration by a questionnaire. In fact, the FSDC is a version of the ACR 2010 criteria for use in survey research. The FSDC were validated in a German cross-sectional survey with 1665 FMS-patients including all levels of care and most specialties involved in the care of FMS-patients (7).

A systematic search of the literature (Cochrane Library, Medline, PsyCInfo and Scopus) from inception to December 2010 was performed for the German interdisciplinary evidence-based guidelines on FMS. Levels of evidence were assigned according to the classification system of the Oxford-Centre for Evidence Based Medicine. The strength of recommendations was built by multiple step formal procedures to reach a consensus. The guidelines were reviewed by the board of directors of the societies engaged in the development of the guidelines (8). Recommendations regarding the classification and diagnosis of FMS were based on expert consensus (9).

\section{RESULTS}

\section{Pre-ACR diagnostic criteria}

FM and fatigue-like illnesses were identified as early as the nineteenth century, and sporadic descriptions of FM can be found through the 1960s. The modern concept of FMS arose in the 1970s to characterize a common group of patients, mostly middle-aged women, who had high levels of pain, multiple complaints, sleep disturbance, psychiatric symptoms, and a generally decreased threshold to painful stimuli (2). Such patients are common in general medicine and represent about $2-4 \%$ of the general European population (10). 
The modern construct of FM arose in 1977 from a single article by Smythe and Moldofsky entitled "Two contributions to the understanding of the "fibrositis" syndrome' (11). The authors identified the characteristics of the syndrome, then called 'fibrositis', and proposed criteria based on what they saw as its key features: nonrefreshing sleep and tender points. Tender points were defined as pre-specified points on the body that, in persons with the syndrome, were particularly sensitive to pressure. The presence of 'widespread aching for longer than three months' and 'disturbed sleep with morning fatigue and stiffness' was also a requirement in these criteria. Decreased pain threshold was measured by a count of tender points.

Tender points rapidly became the central diagnostic feature of the syndrome. Smythe and Moldofsky required tenderness at 12 of 14 anatomic sites $(86 \%)$ to be positive for tenderness. In 1981, Yunus et al. introduced a formal set of criteria (4) as opposed to the ad-hoc criteria used by the above authors. They required aching and pain or stiff-

Table I - The 1990 American College of Rheumatology criteria for the fibromyalgia classification (3).

\begin{tabular}{l}
\hline 1. History of widespread pain \\
\hline Definition. Pain is considered widespread when all of the following are present: \\
- pain in the left side of the body, \\
- pain in the right side of the body, \\
- pain above the waist, \\
- pain below the waist. \\
In addition, axial skeletal pain (cervical spine or anterior chest or thoracic spine or \\
low back) must be present. \\
In this definition, shoulder and buttock pain is considered as pain for each involved \\
side. 'Low back' pain is considered lower segment pain. \\
\hline 2. Pain in 11 of 18 tender point sites on digital palpation \\
\hline Definition. Pain on digital palpation must be present at least in 11 of the following \\
18 sites: \\
- Occiput: Bilateral, at the suboccipital muscle insertions. \\
- Low cervical: bilateral, at the anterior aspects of the intertransverse spaces at \\
C5-C7. \\
- Trapezius: bilateral, at the mid-point of the upper border. \\
- Supraspinatus: bilateral, at origins, above the scapula spine near the medial \\
border. \\
- Second rib: bilateral, at the second costochondral junctions, just lateral to the \\
junctions on upper surfaces. \\
- Lateral epicondyle: bilateral, 2 cm distal to the epicondyles. \\
- Gluteal: bilateral, in upper outer quadrants of buttocks in anterior fold of muscle. \\
- Greater trochanter: bilateral, posterior to the trochanteric prominence. \\
- Knee: bilateral, at the medial fat pad proximal to the joint line. \\
Digital palpation should be performed with an approximate force of 4 kg. \\
\hline
\end{tabular}

ness in three anatomical areas for at least 3 months AND the presence of at least five tender points. In addition, patients had to have three of the following symptoms: modulation of symptoms by physical activity, modulation of symptoms by weather factors, aggravation of symptoms by anxiety or stress, poor sleep, general fatigue or tiredness, anxiety, chronic headache, irritable bowel syndrome, subjective swelling, or numbness. If there were only three to four tender points positive, then five of the symptoms from the symptom list were required. With these criteria, there was a relative de-emphasis on reduced pain threshold and a greater emphasis on the importance of symptoms, a set of symptoms that were often considered to be associated with mental illness.

By the late 1980s there were many different formal and ad hoc criteria sets. There was no clear agreement on which tender point sites should be examined or how they should be examined, nor how many sites had to be tender for a positive examination. Similarly, the format and content of symptom questions was unknown. Both in the clinic and in research settings, the reliability and validity of the available criteria was not known.

\section{The 1990 classification criteria}

Based on comparing patients with similar but non-fibromyalgia pain complaints, the ACR-committee found that the presence of widespread pain combined with at least 11 of 18 tender points best separated patients with fibromyalgia and controls, even though some combinations of symptoms (e.g., fatigue, cognitive problems) were not evaluated (3). This occurred because the authors did not recognize the importance of these symptoms at the time of the study. The authors suggested that the presence of 11 of 18 tender points and the simultaneous presence of WSP for at least 3 months should be the classification criteria for FM (3) (Table I).

Initially intended for research purposes, the criteria were later widely used in clinical diagnosis, particularly among rheumatologists; they were also used in basic science 
and clinical studies. The endorsement by the ACR aided in establishing FM as a respectable clinical diagnosis.

\section{Criticism of the 1990 criteria items}

Numerous concerns were raised on the reliability and validity of the tender point examination (TPE) when used for diagnosis of FMS in the disability setting, which led to the recommendation to stop their use in the clinic (12).

a) A standardized manual tender point survey was developed (13), but this protocol was almost never used by rheumatologists in clinical practice, and was used only in a few clinical studies.

b) The tender point count was shown to be influenced by the interaction between patient and examiner and was highly correlated with distress (14).

c) The tender point count was a poor marker of change in clinical studies. d) The reliability and validity of the TP examination outside the context of FMSspecialized rheumatology settings was never tested.

e) FMS is not a disorder diagnosed and treated by rheumatologists exclusively. Patients are also diagnosed and treated by general practitioners, pain specialists, or mental health specialists. TPE is largely ignored in these settings. Nonrheumatologists had not been trained for TPE within their residency program. Moreover, TPE would be time consuming in these settings. Even if a competent physician of whatever discipline who is able to conduct a thorough medical examination could be taught a standardized manual TPE, the time to carry out this examination could be used to extract a more comprehensive psychosocial history.

f) Although increased tenderness or hy-

Table II - The American College of Rheumatology 2010 preliminary diagnostic criteria for fibromyalgia (5).

\begin{tabular}{l} 
Criteria: \\
\hline A patient satisfies diagnostic criteria for fibromyalgia if the following three conditions are met: \\
1) Widespread Pain Index $>=7$ and Symptom Severity Score $>=5$ or Widespread Pain Index between 3 and 6 and \\
Symptom Severity Score $>=9$ \\
2) Symptoms have been present at a similar level for at least 3 months \\
3) The patient does not have a disorder that would otherwise explain the pain \\
\hline Ascertainment: \\
1) Widespread Pain Index (WPI): Note the number areas in which the patient has had pain over the last week. \\
In how many areas has the patient had pain? Score will be between 0 and 19: \\
Shoulder girdle, Lt Hip (buttock, trochanter), Lt Jaw, Lt Upper back \\
Shoulder girdle, Rt Hip (buttock, trochanter), Rt Jaw, Rt Lower back \\
Upper arm, Lt Upper leg, Lt Chest Neck \\
Upper arm, Rt Upper leg, Rt Abdomen \\
Lower arm, Lt Lower leg, Lt \\
Lower arm, Rt Lower leg, Rt \\
2) Symptom Severity Score: \\
Fatigue \\
Waking unrefreshed \\
Cognitive symptoms \\
For the each of the three symptoms above, indicate the level of severity over the past week using the following \\
scale: \\
$0=$ No problem \\
1 = Slight or mild problems: generally mild or intermittent \\
2 = Moderate: considerable problems, often present and/or at a moderate level \\
$3=$ Severe: pervasive, continuous, life-disturbing problems \\
Considering somatic symptomsa in general, indicate whether the patient has: \\
$0=$ No symptoms \\
1 = Few symptoms \\
2 = A moderate number \\
$3=$ A great deal of symptoms \\
The Symptom Severity Score is the sum of the severity of the three symptoms (fatigue, waking unrefreshed, cognitive \\
symptoms) plus the extent (severity) of somatic symptoms in general. The final score is between 0 and 12. \\
\end{tabular}


peralgesia/allodynia to pressure stimuli had been replicated by other more objective ways of assessment, its relevance and specificity for the diagnosis of FMS had been questioned (15).

g) Chronic widespread pain (CWP) and tender points do not capture other key symptoms such as fatigue and sleeping problems (16).

\section{American College of Rheumatology 2010 preliminary diagnostic criteria}

The 2010 criteria (Table II) addressed a number of problems with the 1990 criteria. They eliminated the TPE, a physical examination item, replaced by the widespread pain index (WPI), a 0-19 count of the number of body regions reported as painful by the patient. In addition, the 2010 criteria assessed on a 0-3 severity scale a series of symptoms that were characteristic of fibro- myalgia: fatigue, non-refreshed sleep, $\operatorname{cog}$ nitive problems, and the extent of somatic symptom reporting. The items were combined into a 0-12 Symptom Severity (SS) scale. Finally, the Widespread Pain Index (WPI) and SS-scales could be combined into a 0-31 fibromyalgianess scale, a second measure of polysymptomatic distress or FM severity (3).

The criteria committee considered that the diagnosis of a symptom severity disorder should require more than an 'augenblick' diagnosis. Instructions, a criteria worksheet, and a patient pain location report are available on-line as an aid to ACR2010 assessments (www.arthritis-research.org/ research/fibromyalgia-criteria).

\section{Survey diagnostic criteria}

The 2010 criteria imposed some burdens on the examiner. The SS-scale items re-

Table III - Fibromyalgia survey questionnaire to assess the Fibromyalgia Diagnostic Survey Criteria (6).

\begin{tabular}{|c|c|c|c|c|}
\hline \multicolumn{5}{|c|}{$\begin{array}{l}\text { I. Using the following scale, indicate for each item the level of severity over } \\
\text { appropriate box. }\end{array}$} \\
\hline \multicolumn{5}{|c|}{$\begin{array}{l}\text { 0: No problem } \\
\text { 1: Slight or mild problems; generally mild or intermittent } \\
\text { 2: Moderate; considerable problems; often present and/or at a moderate level } \\
\text { 3. Severe: continuous, life-disturbing problems }\end{array}$} \\
\hline $\begin{array}{l}\text { Fatigue } \\
\text { Trouble thinking or remembering } \\
\text { Waking up tired (unrefreshed) }\end{array}$ & $\begin{array}{l}\square 0 \\
\square 0 \\
\square 0\end{array}$ & $\begin{array}{l}\square 1 \\
\square 1 \\
\square 1\end{array}$ & $\begin{array}{l}\square 2 \\
\square 2 \\
\square 2\end{array}$ & $\begin{array}{l}\square 3 \\
\square 3 \\
\square 3\end{array}$ \\
\hline \multicolumn{5}{|c|}{ II. During the past 6 months have you had any of the following symptoms? } \\
\hline $\begin{array}{l}\text { Pain or cramps in lower abdomen: } \\
\text { Depression: } \\
\text { Headache: }\end{array}$ & \multicolumn{2}{|c|}{$\begin{array}{l}\square \text { Yes } \\
\square \text { Yes } \\
\square \text { Yes }\end{array}$} & \multicolumn{2}{|c|}{$\begin{array}{l}\square \text { No } \\
\square \text { No } \\
\square \text { No }\end{array}$} \\
\hline \multicolumn{5}{|l|}{ Joint/body pain } \\
\hline \multicolumn{5}{|c|}{$\begin{array}{l}\text { Please indicate below if you have had pain or tenderness over the past } 7 \text { days in each of the areas listed below. } \\
\text { Please make an X in the box if you have had pain or tenderness. Be sure to mark both right side and left side } \\
\text { separately }\end{array}$} \\
\hline $\begin{array}{l}\square \text { Shoulder, left } \\
\square \text { Shoulder, right }\end{array}$ & \multicolumn{2}{|c|}{$\begin{array}{l}\square \text { Upper leg, left } \\
\square \text { Upper leg, right }\end{array}$} & \multicolumn{2}{|c|}{$\begin{array}{l}\square \text { Lower back } \\
\square \text { Upper back } \\
\square \text { Neck }\end{array}$} \\
\hline $\begin{array}{l}\square \text { Hip, left } \\
\square \text { Hip, right }\end{array}$ & \multicolumn{2}{|c|}{$\begin{array}{l}\square \text { Lower leg, left } \\
\square \text { Lower leg, right }\end{array}$} & & \\
\hline $\begin{array}{l}\square \text { Upper am, left } \\
\square \text { Upper arm, right }\end{array}$ & \multicolumn{2}{|c|}{$\begin{array}{l}\square \text { Jaw, left } \\
\square \text { Jaw, right }\end{array}$} & \multicolumn{2}{|c|}{$\begin{array}{l}\square \text { No pain in any of these } \\
\text { areas }\end{array}$} \\
\hline $\begin{array}{l}\square \text { Lower rarm, left } \\
\square \text { Lower arm, right }\end{array}$ & \multicolumn{2}{|c|}{$\begin{array}{l}\square \text { Chest } \\
\square \text { Abdomen }\end{array}$} & & \\
\hline \multicolumn{5}{|c|}{ IV. Overall, were the symptoms listed in I - III above generally present for at least 3 months? } \\
\hline & \multicolumn{2}{|c|}{$\square$ Yes } & \multicolumn{2}{|c|}{$\square$ No } \\
\hline
\end{tabular}


quire a detailed and thoughtful interview of the patient, and the WPI also requires a detailed assessment. Symptom assessment by physicians is inherently subjective. The committee realized that validated questionnaires were available to assess these symptoms. The Fibromyalgia Survey Questionnaire (FSQ) assessing the Fibromyalgia Survey Diagnostic Criteria (FSDC) (Table III) was developed for the assessment of the key symptoms of FMS in survey research and settings where the use of interviews to evaluate the number of pain sites and extent of somatic symptom intensity would be difficult. By substituting a count of three symptoms for the physician's (0-3) evaluation of the extent of somatic symptom intensity by a questionnaire assessing the number of pain sites and somatic symptom severity. Patients who satisfy FSDC meet the following 3 conditions:

1. Widespread Pain Index (WPI) $\geq 7 / 19$ pain sites and Symptom Severity Score (SSS) $\geq 5 / 12$ or WPI between $3-6 / 19$ and $\mathrm{SSS} \geq 9 / 12$;

2. symptoms have been present at a similar level for at least 3 months;

3 . the patient does not have another disorder that would otherwise sufficiently explain the pain (3).

The first two conditions can be assessed by the Fibromyalgia survey questionnaire (FSQ) including the WPI and SSS (6).

The FSQ can be used to assist medical diagnosis. The interpretation and assessment of questionnaire's validity, however, must be the work of the physician. Selfdiagnosis of FMS based only on the FSQ is not allowed. The use of questionnaires for screening purposes and assisting diagnoses is common in psychiatry, psychology and pain medicine, and can be applied to primary care, too.

\section{OPEN QUESTIONS}

Fibromyalgia or fibromyalgia syndrome? The endorsement of the classification criteria of "fibromyalgia" by the ACR brought official recognition to the term. "Fibromyalgia" received an International Classifica- tion of Disease (ICD) code by the World Health Organization and is included into the diseases of the (ICD M 79.7). "Fibromyalgia" is listed in the International Classification of Diseases of the World Health Organisation within Chapter M "Diseases of the musculoskeletal system and connective tissue" with the code M 79.7 (17).

The German guideline recommends the use of the term "fibromyalgia syndrome" rather than "fibromyalgia" to preclude confusion with a distinct somatic (rheumatology) disease (9), as there is no clear evidence of a structural organ damage underlying FMS-symptoms (18). A syndrome is defined to be a group of symptoms that collectively indicate or characterize a disease, psychological disorder, or other abnormal condition. Based on the currently available evidence, FMS is classified to be a first rank syndrome (unknown etiology, heterogenous pathogenesis and defined phenotype) (19). FMS may be classified as a functional somatic syndrome - defined by a typical group of symptoms and the exclusion of a somatic disease sufficiently explaining the symptoms (9).

\section{How to assess CWP?}

Despite all controversies, there is an agreement between most clinicians, that CWP is one main clinical feature of FMS. In the clinics, CWP can be assessed by a pain diagram (blank body manikin) filled out by the patient (Figures 1 and 2). For the busy clinician, the most frequently used definitions of CWP can be cumbersome to use. The ACR 1990 criteria define CWP to be pain in the left side of the body, pain in the right side of the body, pain above the waist, and pain below the waist; axial skeletal pain (cervical spine or anterior chest or thoracic spine or low back). In this definition, shoulder and buttock pain is considered as pain for each involved side. 'Low back' pain is considered lower segment pain (5).

Other criteria for widespread pain (WSP) have been proposed. The more complex Manchester criteria require pain in at least two sections of two contralateral limbs and in the axial skeleton. Pain must be present 


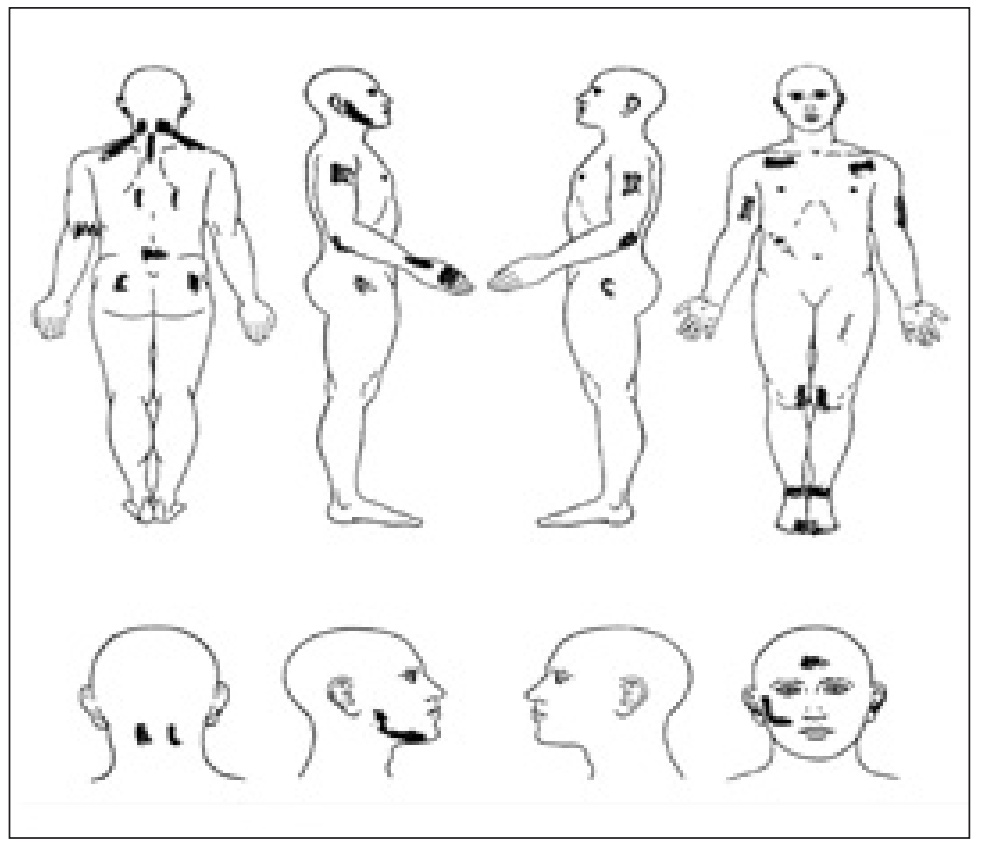

Figure 1 - Pain diagram from a female patient diagnosed with fibromyalgia syndrome.

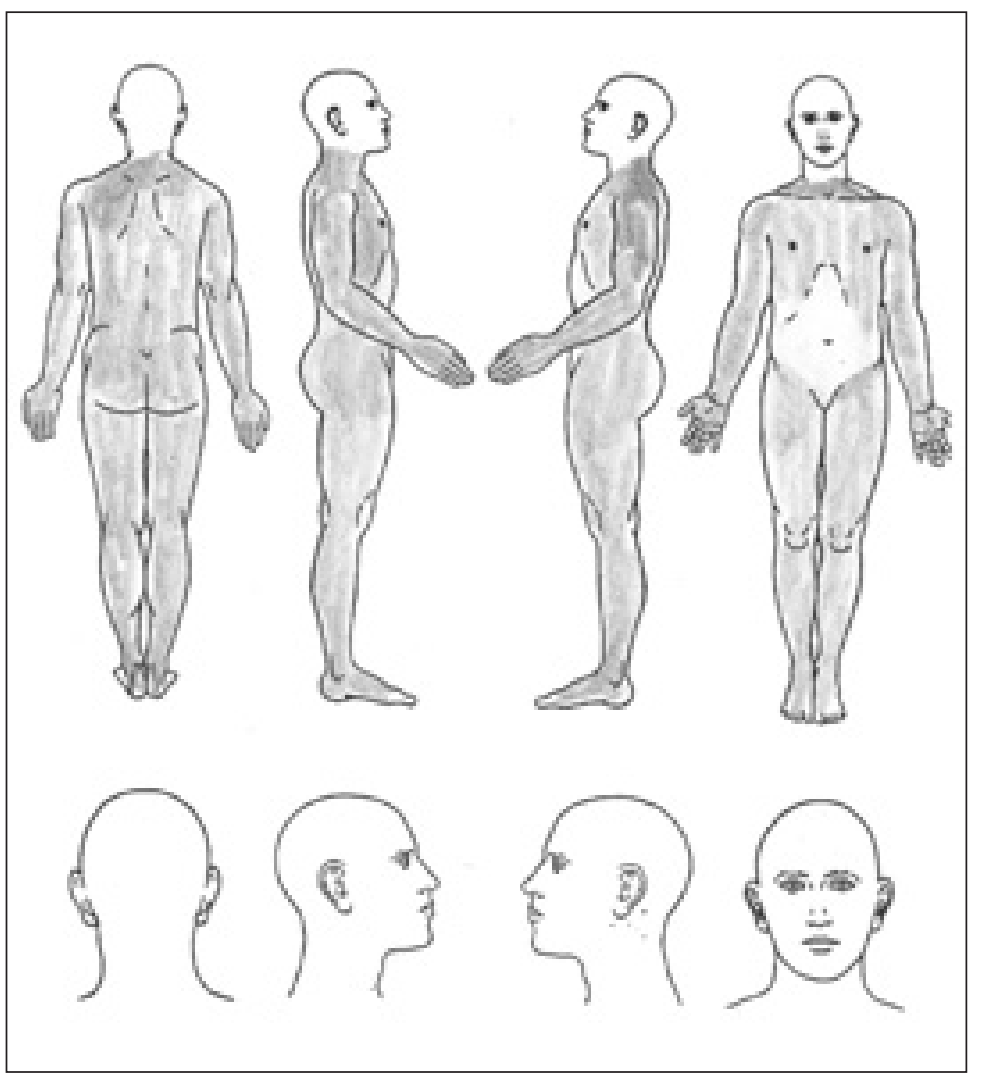

Figure 2 - "All over" pain diagram from a female patient diagnosed with fibromyalgia syndrome. in two separate sections of a body quadrant to be positive (20).

The criteria of pain in 3 or more segments of the body quadrant (21) or of pain axially and in all 4 extremities (22) are more intuitive. Many patients report and mark a pain "all over" in the manikin (Figure 2). In these cases, every criterion of CWP is met. Alternatively, the patient can complete the WPI. WSP is defined by $\geq 7 / 19$ pain sites (6). In a German multicenter study including different specialties the concordance rate between the ACR 1990 and WPI-criteria of WP ( $\geq 7 / 19$ pain sites) was $100 \%$ (22). The important point at a clinical level is to identify patients with CWP.

\section{Different diagnostic criteria for FMS - does it matter?}

The concordance rate of the ACR 1990 classification criteria with the "Katz" criteria, an ancestor of the FSDC, was $72 \%$ in an US American rheumatology practice (23) and $80 \%$ in a German study including different specialties (21). The concordance rate between the ACR 1990 and FSDC criteria was $73 \%$ in subsample of 128 patients (7).

As with CWP-definitions, patients with high levels of polysymptomatic distress (high levels of bodily and psychological symptoms) will meet all FMS-criteria. Whether the preliminary ACR 2010 or the FSDC - criteria will lead to change in the prevalence of FMS or to a change of the clinical features of the FMS-populations still needs to be determined. Preliminary data suggest that FMS may be more frequently diagnosed in men by the new diagnostic criteria than the old, because they tender point criterion was one reason which led to a preponderance of women in the FMS-population (24). In CWP, most epidemiology studies found an equal gender distribution (25).

\section{Incomplete FMS?}

Whatever diagnostic criteria are used, there will always be some patients left who do not meet the "FMS-positive" criterion at the time of the examination. In the ACR 2010 criteria study, $25 \%$ of previously di- 
agnosed FMS patients did not meet criteria (5). In a single center study of an US rheumatology clinic, $18.7 \%$ of the patients previously diagnosed with FMS did not meet the ACR 1990 criteria of FMS. These patients were labeled "incomplete FMS (IFMS)". IFMS-patients reported less frequent and severe symptoms than FMSpatients (26). This study highlights the usefulness of conceptualizing FMS to be the end of the spectrum of biopsychosocial distress $(14,27,28)$, and to use continuous scales such as the WPI and SSS to assess symptom-burden in patients instead of classifying patients FMS-positive or negative. Using the IFMS definition, potentially everyone has IFMS.

\section{Assessing the severity of FMS}

While the ACR 2010 criteria case definition of fibromyalgia implies a general homogeneity in core symptoms (degree of WSP, fatigue, cognitive, sleep problems, and excess somatic symptom reporting), FMS remains a complex of various symptoms: Patients differ in the amount of somatic and psychological symptoms, disability, coping and associated psychosocial problems (17). Generally accepted severities of FMS are not available. Approaches to grading fibromyalgia severity include use the ACR 2010 Symptom Severity Scale (3) and the Polysymptomatic Distress Scale (or Fibromyalgianess Scale) (6).

The Fibromyalgia Impact Questionnaire (FIQ)-Revised total scores (29) has been used to define FM severity, with $0-<39$, 39$<59$, and 59-100, representing mild, moderate, and severe FM, respectively (30).

\section{FMS and somatic disease?}

The ACR 1990 classification study concluded that the diagnosis of FMS can be accomplished with confidence even in the presence of other medical conditions (3). Most notably the "other medical conditions" assessed in this study were rheumatic diseases such as rheumatoid arthritis, osteoarthritis, and low back and neck pain syndromes, but not other internal or neurology diseases which can present with widespread pain. The study results have not been tested in such settings. The prevalence-rates of FMS in diseases with structural (organic) damages had been found to range between $12-20 \%$ in rheumatoid arthritis, 5-25\% in Lupus erythematosus, 11$50 \%$ in ankylosing spondylitis, $17-18 \%$ in diabetes mellitus and 3-49\% in inflammatory bowel diseases (31). In fact, in every somatic disease patients with high levels of psychosocial distress which can be labelled "FM" can be identified (32).

The preliminary ACR 2010 criteria require that the fibromyalgia diagnosis be excluded in the presence of a somatic disease which could sufficiently explain the symptoms (5). Not infrequently FMS occurs in patients with other diseases. In such a situation the physician can diagnose both disorders. A problem arises when the "other" disease has signs and/or symptoms similar to those found in FMS, leading to believe that FMS is present when it is not. A patient with extensive burns would have many tender points and WSP. Though satisfying ACR 1990 criteria, we would not be able to diagnose FMS in such a patient. The situation is more complex (and realistic) when we consider diseases like metastatic cancer. In such a situation the patient might satisfy FMS criteria by virtue of cancer or of FMS, or of both. A comprehensive history, physical examination and appropriate screening test most often would clarify the issue. The physician could choose to diagnose one or both disorders, depending on clinical judgment.

In population studies that use the FDSC the existence of other diseases may not be known. Because of this, FMS could be over-diagnosed and the apparent prevalence inflated. But such disorders are uncommon. Their actual importance would depend on the prevalence of the underlying disorder multiplied by the proportion with that disorder which has fibromyalgia signs and symptoms caused only by that disorder. The net effect would be very small.

\section{Do we need FMS-screening tools?}

In our opinion, no FMS-screening tools are necessary in general medicine, rheumatology and pain medicine practice. In these 
Table IV - Some differential diagnoses of chronic widespread pain (35).

\begin{tabular}{|l|l|}
\hline Internal diseases & Neurology diseases \\
\hline Chronic inflammatory rheumatic disease & Inflammatory myopathies \\
\hline Chronic hepatitis C & Metabolic myopathies \\
\hline Chronic inflammatory bowel disease & Degenerative myopathies \\
\hline Celiac disease & Endocrine myopathies \\
\hline Osteoporosis & Myotonia \\
\hline Hyper-/Hypoparathyreoidism & Toxic myalgias \\
\hline Hyper-/Hyopthyreosis & Myalgias in rare disease (e.g., Stiff Person Syndrome) \\
\hline Vitamin D deficiency & Myalgias with lesions of the central and peripheral nervous system \\
\hline
\end{tabular}

settings patients with chronic pain should be assessed for CWP. If in case of CWP symptom questionnaires are used to capture FMS-symptoms, we recommend the FSQ (7), because its scores can be used (together with the medical assessment) for the clinical diagnosis.

The following screening tools had been developed and might be useful for screening in survey research or other settings (e.g., psychiatry):

1. The Fibromyalgia Rapid Screening Tool (FiRST) was tested in a prospective French multicenter study of 162 patients with chronic pain due to FM (according to ACR 1990 criteria) $(n=92)$ compared with a group of patients with chronic diffuse pain due to other rheumatic conditions, including rheumatoid arthritis $(n=32)$, ankylosing spondylitis $(n=25)$ and osteoarthritis $(n=13)$. A cut-off score of 5 (corresponding to the number of positive items with a highest score of 6) gave the highest rate of correct identification of patients $(87.9 \%)$, with a sensitivity of $90.5 \%$ and a specificity of $85.7 \%$ (33).

2. The Fibromyalgia Diagnostic Screen was developed for use by primary care clinicians to assist in the diagnostic evaluation of FM (34).

Recommendation of the german guidelines on fms for an initial diagnostic workup for patients with chronic widespread pain in primary and specialist care

\section{History taking}

Patients presenting with chronic pain (most days within the last 3 months) should be screened for CWP by filling out a pain diagram or the WPI. If CWP is diagnosed, other key symptoms of FMS, fatigue and non-restorative sleep should be explored. The symptoms can be documented by the ACR 2010 Symptom Severity Scale or the FSQ. Finally all types of medication used by the patient should be assessed since arthralgia, myalgia and fatigue can also be side effects of medication (Table IV).

\section{Physical examination}

The patients suspected of having FMS should have a complete physical examination, including an orthopedic and neurological examination.

\section{Blood tests and diagnostic imaging}

Numerous somatic diseases can lead to CWP, fatigue and sleep disturbances (35) (Table V) and that these symptoms can be relieved by medical treatment.

The following routine blood tests are recommended for patients with CWP (potential differential diagnosis or concomitant diagnoses are indicated in brackets):

a) Blood sedimentation rate, C-reactive protein, red and white cell blood count (polymyalgia rheumatica, rheumatoid arthritis).

b) Creatinin kinase (muscle disease).

c) Calcium (hypercalcaemia).

d) Thyroid-stimulating hormone (hypothyroidism).

e) Depending on history and examination further blood tests can be necessary if other differential diagnoses are suspected.

Without clinical signs, routine testing for antibodies associated with rheumatoid dis- 
Table V - Drug induced myopathies (35).

\begin{tabular}{|l|l|l|}
\hline Inflammatory myopathies & Other myopathies & Myopathies and neuropathies \\
\hline Cimetidine & Aromatase inhibitors & Amiodarone \\
\hline D-Penicillamine & Carbimazole & Colchicine \\
\hline Cocaine & Clofibrate & Heroine \\
\hline Levodopa & Cromoglycin acid & Interferon \\
\hline Penicilline & Cyclosporine & L-Tryptophane \\
\hline Procainamide & Enalaprile & Vincristine \\
\hline Sulfonamide & Ezitimibe & \\
\hline Zidovudine & HMG-CoA reductase inhibitor & \\
\hline & Metoprolole & \\
\hline & Minoxidile & \\
\hline & Proton pump inhibitor & \\
\hline & Salbutamole & \\
\hline
\end{tabular}

eases or lupus is not recommended. If no other diseases are suspected on clinical grounds, including those that require imaging studies for diagnosis (e.g., joint diseases), X-rays or other diagnostic imaging studies are not recommended.

\section{Referral to specialists}

In case of a suspected internal, neurological or orthopaedic disease (partially explaining the symptoms) referral to a specialist is recommended. Tests profiles for concomitant internal diseases have been described (36).

\section{Final diagnosis}

In a report, physicians should detail the criteria used (ACR 1990, ACR 2010, FSDC). If FMS-diagnosis is established, patients should be screened for symptoms of other functional somatic syndromes, e.g. irritable bowel syndrome. Moreover restrictions of daily activities due to the symptoms should be explored. Patients should be asked about current psychosocial problems (job, personal relations) and screened for anxiety and depression.

The screening questions for depression and anxiety of the Patient Health Questionnaire PHQ 4 (37) are recommended:

Over the last 2 weeks, have you been bothered by the following problems?

- Feeling nervous, anxious or on edge (Anxiety).

- Not being able to stop or control worrying (Anxiety).
- Little interest or pleasure in doing things (Depression).

- Feeling down, depressed, or hopeless (Depression).

The amount of additional somatic and psychological symptoms, psychosocial problems and limitations of daily activities can help to grade the severity of FMS.

Patients with positive screens for anxiety and/or depression, severe restrictions of daily functions and severe psychosocial problems should be referred to a metal health specialist (psychosomatic medicine, clinical psychology, psychiatry) (38).

\section{CONCLUSIONS}

Should rheumatologists by the primary physician for FMS (39)? A survey of 600 patients in six European countries, South Korea and Mexico demonstrated that the specialties initially contacted for FMSsymptoms and for long-term treatment were quite variable across countries (40).

The new diagnostic criteria do not require a rheumatologist to perform TPE. The diagnosis of FMS is easy to establish in most patients with CWP and no signs of a somatic disease as cause of CWP. A rheumatologist's expertise might be needed to exclude difficult to diagnose or concomitant inflammatory rheumatic diseases. Given the high rate of comorbid functional somatic syndromes and mental disorders in FMS (41), a mental health specialist's evaluation 
is recommended in the case of a suspected mental disorder when diagnosis is uncertain to the primary care physician, where maladaptive coping (e.g., fear-avoidance behaviour) is present, or there are current severe psychosocial conflicts (38). By which specialties FMS-patients will be treated in the long-term, it can depend on the type of health care system and the individual preferences of the physicians.

\section{REFERENCES}

1. Wolfe F. Fibromyalgia wars. J Rheumatol. 2009; 36: 671-8.

2. Wolfe F, Häuser W. Fibromyalgia diagnosis and diagnostic criteria. Ann Med. 2011; 43: 495-502.

3. Wolfe F, Smythe HA, Yunus MB, et al. The American College of Rheumatology 1990 Criteria for the Classification of Fibromyalgia. Report of the Multicenter Criteria Committee. Arthritis Rheum. 1990; 33: 160-72.

4. Yunus M, Masi AT, Calabro JJ, et al. Primary fibromyalgia (fibrositis): clinical study of 50 patients with matched normal controls. Semin Arthritis Rheum 1981; 11: 151-71.

5. Wolfe F, Clauw DJ, Fitzcharles MA, et al. The American College of Rheumatology preliminary diagnostic criteria for fibromyalgia and measurement of symptom severity. Arthritis Care Res (Hoboken). 2010; 62: 600-10.

6. Wolfe F, Clauw DJ, Fitzcharles MA, et al. Fibromyalgia criteria and severity scales for clinical and epidemiological studies: a modification of the ACR Preliminary Diagnostic Criteria for Fibromyalgia. J Rheumatol. 2011; 38: 1113-22.

7. Häuser W, Jung E, Erbslöh-Möller BF, et al. Validation of the fibromyalgia survey questionnaire within a cross-sectional survey. PLOS One. 2012; 7: e37504.

8. Häuser W, Bernardy K, Wang H , Kopp I. Reports of methods. Schmerz 2012; 26: in press

9. Eich W, Häuser W, Arnold B, et al. Definition, classification, clinical diagnosis and prognosis of fibromyalgia syndrome. Schmerz. 2012; 26: in press.

10. Branco JC, Bannwarth B, Failde I, Abello Carbonell J, Blotman F, Spaeth M, et al.Prevalence of fibromyalgia: a survey in five European countries. Semin Arthritis Rheum. 2010; 39: 448-53.

11. Smythe HA, Moldofsky H. Two contributions to under 13. standing of the 'fibrositis' syndrome. Bull Rheum Dis. 1977; 28: 928-31.

12. Wolfe F. Stop using the American College of Rheumatology criteria in the clinic. J Rheumatol. 2003; 30: 1671-2.
13. Okifuji A, Turk DC, Sinclair JD, Starz TW, Marcus DA. A standardized manual tender point survey. I. Development and determination of a threshold point for the identification of positive tender points in fibromyalgia syndrome. J Rheumatol. 1997; 24: 377-83.

14. Wolfe F. The relation between tender points and fibromyalgia symptom variables: evidence that fibromyalgia is not a discrete disorder in the clinic. Ann Rheum Dis. 1997; 56: 268-71.

15. Gracely RH. A pain psychologist's view of tenderness in fibromyalgia. J Rheumatol. 2007; 34: 912-3.

16. Turk DC, Flor H. Primary fibromyalgia is greater than tender points: toward a multiaxial taxonomy. J Rheumatol. 1989; 19: 80-6.

17. World Health Organisation. International Classification of Diseases Version 2007. Available from: www.who.int/classifications/ apps/icd/icd10online/?gm70.htm+m797

18. Sommer C, Häuser W, Burgmer M, et al. Etiology and pathophysiology of fibromyalgia syndrome. Schmerz. 2012; 26: in press.

19. Leiber B. Die klinischen Syndrome. 1990, Stuttgart, Urban \& Schwarzenberg.

20. Hunt IM, Silman AJ, Benjamin S, et al. The prevalence and associated features of chronic widespread pain in the community using the "Manchester" definition of chronic widespread pain. Rheumatology. 1999; 38: 275-9.

21. Bennett R. Fibromyalgia, chronic fatigue syndrome, and myofascial pain. Curr Opin Rheumatol. 1998; 10: 95-103.

22. Häuser W, Hayo S, Biewer W, et al. Diagnosis of fibromyalgia syndrome-a comparison of Association of the Medical Scientific Societies in Germany, survey, and American College of Rheumatology criteria. Clin J Pain. 2010; 26: 505-11.

23. Katz RS, Wolfe F, Michaud K. Fibromyalgia diagnosis: a comparison of clinical, survey, and American College of Rheumatology criteria. Arthritis Rheum. 2006; 5: 169-76.

24. Häuser W, Kühn-Becker H, von Wilmoswky $\mathrm{H}$, et al. Demographic and clinical features of patients with fibromyalgia syndrome of different settings: a gender comparison. Gend Med. 2011; 8: 116-25.

25. Mourão AF, Blyth FM, Branco JC. Generalised musculoskeletal pain syndromes. Best Pract Res Clin Rheumatol. 2010; 24: 829-40.

26. Yunus MB, Aldag JC. The concept of incomplete fibromyalgia syndrome: comparison of incomplete fibromyalgia syndrome with fibromyalgia syndrome by 1990 ACR classification criteria and its implications for newer criteria and clinical practice. J Clin Rheumatol. 2012; 18: 71-5.

27. Croft P, Burt J, Schollum J, Thomas E, Macfarlane G, Silman A. More pain, more tender points: is fibromyalgia just one end of a con- 
tinuous spectrum? Ann Rheum Dis. 1996; 55: 482-5.

28. Häuser W, Schmutzer G, Brähler E, Glaesmer H. A cluster within the continuum of biopsychosocial distress can be labeled "fibromyalgia syndrome" - evidence from a representative German population survey. J Rheumatol. 2009; 36: 2806-12.

29. Bennett RM, Friend R, Jones KD, et al. The Revised Fibromyalgia Impact Questionnaire (FIQR): validation and psychometric properties. Arthritis Res Ther. 2009; 11: R120.

30. Schaefer C, Chandran A, Hufstader M, et al. The comparative burden of mild, moderate and severe Fibromyalgia: results from a crosssectional survey in the United States. Health Qual Life Outcomes. 2011; 9: 71.

31. Yunus MB. The prevalence of fibromyalgia in other chronic pain conditions. Pain Res Treat. 2012; 2012: 584573.

32. Creed FH, Davies I, Jackson J, et al The epidemiology of multiple somatic symptoms. J Psychosom Res. 2012; 72: 311-7.

33. Perrot S, Bouhassira D, Fermanian J. Cercle d'Etude de la Douleur en Rhumatologie. Development and validation of the Fibromyalgia Rapid Screening Tool (FiRST). Pain. 2010; 150: 250-6.

34. Arnold LM, Stanford SB, Welge JA, Crofford
LJ. Development and testing of the fibromyalgia diagnostic screen for primary care. J Womens Health (Larchmt). 2012; 21: 231-9.

35. Köllner V, Bernardy K, Sommer C, Häuser W. [Diagnosis and therapy of fibromyalgia syndrome.] [Article in German]. Dtsch Med Wochenschr. 2009; 134: 1163-74.

36. Russell IJ, Raphael KG. Fibromyalgia syndrome: presentation, diagnosis, differential diagnosis, and vulnerability. CNS Spectr. 2008; 13 (Suppl. 5): 6-11.

37. Kroenke K, Spitzer RL, Jannett BW, et a B. An Ultra-brief screening scale for anxiety and depression: The PHQ-4. Psychosomatics. 2009; 50: 613-21.

38. Köllner V, Häuser W, Bernardy BH, et al. Systemtaic review, meta-analysis and guideline: Psychotherapy in patients with fibromyalgoia syndrome. Schmerz. 2012; 26: in press.

39. Shir Y, Fitzcharles MA. Should rheumatologists retain ownership of fibromyalgia? J Rheumatol. 2009; 36: 667-70.

40. Choy E, Perrot S, Leon T, et al. A patient survey of the impact of fibromyalgia and the journey to diagnosis. BMC Health Serv Res. 2010; 10: 102.

41. Henningsen P, Zimmermann T, Sattel H. Medically unexplained physical symptoms, anxiety, and depression: a meta-analytic review. Psychosom Med. 2003; 65: 528-33. 